Journal of Experimental and Theoretical Physics, 1998, 86 (4): 661-665

\title{
Diffusing-wave spectroscopy in randomly inhomogeneous media with spatially localized scatterer flows
}

\author{
S. E. Skipetrov \\ M. V. Lomonosov Moscow State University, 119899 Moscow, Russia \\ I. V. Meglinski \\ N. G. Chernyshevskiŭ Saratov State University, 410026 Saratov, Russia
}

\begin{abstract}
Multiple scattering of laser radiation in a randomly inhomogeneous turbid medium with a spatially localized flow of particles is studied. The time autocorrelation function of backscattered light is calculated for the case of a laminar flow of scatterers in a cylindrical capillary embedded in the medium. A new method is proposed and tested experimentally for determining the position of the dynamic region and the dominant form and characteristic velocity of the particle motion there.
\end{abstract}

\section{INTRODUCTION}

Problems involving the multiple-scattering of light in randomly inhomogeneous media have been attracting a great deal of attention in recent years. ${ }^{1}$ This is due to both the diversity of beautiful physical effects observed under multiple-scattering conditions (coherent backscattering ${ }^{2,3}$ and angular and temporal correlations of the scattered radiation ${ }^{4}$ ) and, in connection with the extensive use of optical diagnostics methods in modern medicine, ${ }^{5-7}$ the extreme importance of correctly describing the processes occurring under these conditions.

So-called diffusing-wave spectroscopy has been developing rapidly during the last ten years. Its foundations were laid in Refs. 8 and 9. The method of diffusing-wave spectroscopy is based on measuring the time autocorrelation function $G_{1}(\tau)=\left\langle E(t) E^{*}(t-\tau)\right\rangle$ of light that is multiply scattered in a turbid medium. It is found that even under the conditions of strong multiple scattering $G_{1}(\tau)$ is sensitive to the character and intensity of scatterer motion in the medium. Moreover, by measuring $G_{1}(\tau)$ it is possible to detect very small displacements of the light-scattering particles (up to hundredths of a wavelength $\lambda$ of the radiation employed), while the methods based on measuring the characteristics of single scattering of waves are sensitive only to scatterer displacements over a distance of the order of $\lambda .{ }^{9}$ Another interesting possible application of diffusing-wave spectroscopy has been discovered in the last few years - the possibility of determining the location and performing diagnostics of dynamic inhomogeneities in turbid media on the basis of an analysis of the scattered radiation. ${ }^{10-13}$

In the present paper we propose a method of determining the location and measuring the characteristics of directed scatterer flows which are hidden deep in a turbid medium. The method makes it possible to obtain information about the motion of particles inside a medium on the basis of the dependence $G_{1}(\tau)$ measured at different points on the surface of the sample. The theoretical and experimental results presented are in good agreement with one another. Moreover, they agree with both the results of other experiments ${ }^{13}$ and theoretical calculations. ${ }^{12}$ The potential possibilities and limitations of the method of measuring the characteristics of scatterer flows in strongly light-scattering turbid media are evaluated. The method we propose could find interesting applications, for example, in hemodynamics, since it opens up the possibility of performing noninvasive measurements of the velocity of blood flow in blood vessels and detecting changes in blood volume in capillary ansae and other biological tissues. ${ }^{14}$

\section{THEORETICAL ANALYSIS}

Let us consider the scattering of light with wavelength $\lambda$ in a sample of a turbid medium (particle size $\sim \lambda$ ), characterized by a photon transport mean-free path $\ell^{*}=\left(\mu_{s}^{\prime}\right.$ $\left.+\mu_{a}\right)^{-1}$, where $\mu_{s}^{\prime}$ and $\mu_{a}$ are the scattering and absorption coefficients, ${ }^{15}$ and in addition $\mu_{a} \ll \mu_{s}^{\prime}$. Under strong multiple-scattering conditions $\left(\lambda \ll \ell^{*} \ll L\right.$, where $L$ is the characteristic size of the sample) the time correlation function $G_{1}(\mathbf{r}, \tau)=\left\langle E(\mathbf{r}, t) E^{*}(\mathbf{r}, t-\tau)\right\rangle$ of depolarized multiplyscattered radiation measured at point $\mathbf{r}$ on the boundary $S$ of the sample can be described in the diffusion approximation by solving the stationary diffusion equation ${ }^{10,13}$

$$
\left[\nabla^{2}-\alpha^{2}(\tau)\right] G_{1}(\mathbf{r}, \tau)=-\frac{F(\mathbf{r})}{D_{p}}
$$

with the boundary condition ${ }^{16}$

$$
G_{1}(\mathbf{r}, \tau)-\frac{2}{3} \ell^{*}\left(\mathbf{n} \cdot \nabla G_{1}(\mathbf{r}, \tau)\right)=0, \quad \mathbf{r} \in S .
$$

Here we have neglected light absorption in the medium, $F(\mathbf{r})$ describes the distribution of light sources, $D_{p}=c \ell^{*} / 3$ is the light diffusion coefficient in the medium, ${ }^{15} \mathbf{n}$ is the inward unit normal to the surface $S$, and the specific form of the function $\alpha(\tau)$ depends on the character of the scatterer motion in the medium: $\alpha^{2}(\tau)=3 \tau / 2 \tau_{0} \ell^{* 2}$ in the case of 


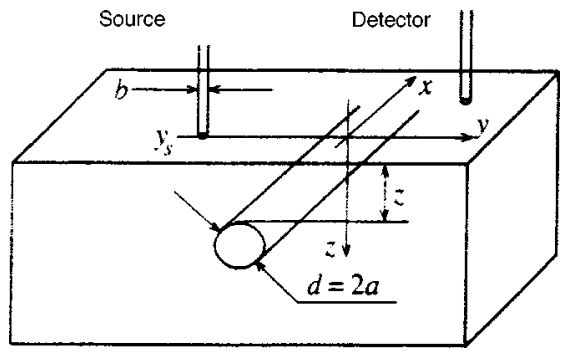

FIG. 1. Schematic diagram of the experiment. The sample $\left(8 \times 15 \times 15 \mathrm{~cm}^{3}\right)$ consists of particles of rutile $\left(\mathrm{TiO}_{2}\right)$ suspended in resin $(0.01 \mathrm{~g}$ of rutile per $100 \mathrm{ml}$ of resin, particle diameter $0.25 \mu \mathrm{m}, \mu_{s}^{\prime}=4 \mathrm{~cm}^{-1}, \mu_{a}=0.002 \mathrm{~cm}^{-1}$, $\left.l^{*}=0.25 \mathrm{~cm}, D_{B}=0\right)$. A transverse cylindrical opening with diameter $d$ $=0.75 \mathrm{~cm}$, where a flow of a suspension of polystyrene beads in water (particle diameter $0.296 \mu \mathrm{m}$, concentration $\approx 0.5 \%, D_{B}=1.5 \times 10^{-8} \mathrm{~cm}^{2} / \mathrm{s}$, $\tau_{0}=6.32 \times 10^{-4} \mathrm{~s}$ ) is produced, was made parallel to the surface inside the sample at a depth $z=0.925 \mathrm{~cm}$. Laser radiation is introduced into the medium by means of a narrow fiber-optic lightguide with diameter $b<\ell^{*}$. The time autocorrelation function of the intensity of the multiply scattered light in the medium is measured at the point $(x, y, 0)$.

Brownian motion of particles in a medium with diffusion coefficient $D_{B},{ }^{9}$ where $\tau_{0}=\left(4 k^{2} D_{B}\right)^{-1}$ and $k=2 \pi / \lambda$, and $\alpha^{2}(\tau)=6\left(\tau / \tau_{f} \ell^{*}\right)^{2}$ in the case of laminar scatterer flow, ${ }^{17}$ where the characteristic time $\tau_{f}$ depends on the flow geometry. In the case when the directed motion of the lightscattering particles is superimposed on the random walk of the particles, $\alpha^{2}(\tau)$ is given by a sum of terms corresponding to these two different types of motion. ${ }^{18}$

The boundary condition (2) can be approximately replaced by requiring $G_{1}(\mathbf{r}, \tau)$ to vanish on the so-called extrapolated boundary ${ }^{15} z=-z_{1}=-\Delta \ell^{*}$, where $\Delta$ depends on the scattering conditions near the boundary. When the scattering is isotropic and the refractive index of the scattering medium equals that of the surrounding medium, the Milne theory gives $\Delta=0.7104{ }^{19}$

Let the turbid medium fill the half-space $z>0$ and let the medium contain a hidden inclusion in the form of a cylindrical capillary with diameter $d=2 a>\ell^{*}$. Let the capillary be directed along the $x$ axis and located at a distance $z$ from the boundary of the medium (Fig. 1). We denote by $S_{1}$ the surface of the capillary and by $V_{1}$ the volume enclosed by it. If a directed scatterer flow is produced in the capillary, while elsewhere in the medium the scatterers move as Brownian particles, then we can introduce in Eq. (1) a spatial dependence of $\alpha^{2}(\tau)$ of the form ${ }^{13}$

$$
\alpha^{2}(\tau)= \begin{cases}\alpha_{\text {in }}^{2}(\tau)=3 \tau / 2 \tau_{0} \ell^{* 2}+6\left(\tau / \tau_{f} \ell^{*}\right)^{2}, & \mathbf{r} \in V_{1}, \\ \alpha_{\text {out }}^{2}(\tau)=3 \tau / 2 \tau_{0} \ell^{* 2}, & \mathbf{r} \notin V_{1} .\end{cases}
$$

In our experimental situation (Fig. 1) a Poiseuille velocity profile can be assumed for the particles inside the capillary, which gives

$$
\tau_{f}=\sqrt{30} / k \ell^{*} \Gamma_{1}, \text { where } \Gamma_{1}=32 Q / \sqrt{2} \pi d^{3},
$$

and $Q$ is the volume flow rate of the liquid, equal to the volume of the liquid passing through the cross section of the capillary per unit time. It is easy to show that $Q$ is related to the average velocity $V$ of the directed motion of the particles in the capillary by the relation $Q=\pi a^{2} V$.
Finally, we write the boundary conditions on the surface $S_{1}$ of the capillary in the form ${ }^{10}$

$$
\begin{aligned}
& G_{1}^{\text {in }}(\mathbf{r}, \tau)=G_{1}^{\text {out }}(\mathbf{r}, \tau), \quad \mathbf{r} \in S_{1}, \\
& \left(\mathbf{n} \cdot \nabla G_{1}^{\text {in }}(\mathbf{r}, \tau)\right)=\left(\mathbf{n} \cdot \nabla G_{1}^{\text {out }}(\mathbf{r}, \tau)\right), \quad \mathbf{r} \in S_{1},
\end{aligned}
$$

where $G_{1}^{\text {in,out }}(\mathbf{r}, \tau)$ are solutions of Eq. (1) inside and outside the volume $V_{1}$, respectively.

To complete the mathematical formulation of the problem we have only to specify the distribution $F(\mathbf{r})$ of the light sources in the medium. In an experiment the sample is ordinarily illuminated by a laser beam of finite width $b$. The cases $b \gg \ell^{*}$ and $b<\ell^{*}$ are easiest to describe theoretically, since in the first case one can assume approximately that a plane wave is incident on the surface of the medium, while in the second case one can study a point source of radiation positioned at a point determined by its coordinate $y_{s}$ on the surface of the medium (see Fig. 1). Since the coherent laser radiation becomes diffuse at a depth $z_{0} \sim \ell^{*},{ }^{15}$ we shall write the source function in Eq. (1) in the form

$$
F(\mathbf{r}) \simeq \begin{cases}\delta\left(z-z_{0}\right), & b \gg \ell^{*}, \\ \delta(x) \delta\left(y-y_{s}\right) \delta\left(z-z_{0}\right), & b<\ell^{*} .\end{cases}
$$

On this basis it is easy to obtain an expression for the correlation function $G_{1}^{0}$ of depolarized light backscattered from an infinite medium in the absence of a scatterer flow in the capillary $\left(\tau_{f} \rightarrow \infty\right)$. In the limit $\tau \ll \tau_{0}$ we obtain

$$
G_{1}^{0}(\tau)=\exp \left\{-\gamma \alpha_{\text {out }} \ell^{*}\right),
$$

for $b \gg \ell^{*}$ and

$$
\begin{aligned}
G_{1}^{0}(x, y, \tau)= & \frac{1}{4 \pi D_{p}}\left\{\frac{\exp \left(-\alpha_{\text {out }} \rho\right)}{\rho}\right. \\
& \left.-\frac{\exp \left(-\alpha_{\text {out }} \sqrt{\rho^{2}+4 \gamma^{2} \ell^{* 2}}\right)}{\sqrt{\rho^{2}+4 \gamma^{2} \ell^{* 2}}}\right\}
\end{aligned}
$$

for $b<\ell^{*}$. In these formulas $\gamma=1+\Delta$ is a numerical constant of the order of 2, $\rho=\left[x^{2}+\left(y-y_{s}\right)^{2}\right]^{1 / 2}$ (see Fig. 1), and the light source is assumed to be located at the point $\left(0, y_{s}\right)$ on the surface of the medium. The results (8) and (9) were obtained earlier by different methods, and they have also been confirmed experimentally. ${ }^{8,9}$ We note that the correlation function (8) does not depend on the position $(x, y)$ of the detector on the surface of the medium or on the photon transport mean free path $\ell^{*}$.

Now let a laminar flow of light-scattering particles be produced in the capillary. In this case we write the solution in the form $G_{1}(x, y, \tau)=G_{1}^{0}(x, y, \tau)+G_{1}^{S}(x, y, \tau)$, where the last term describes the effect of the flow on the correlation function. Neglecting the condition (2) on the boundary of the medium, we obtain for the case $b \gg \ell *$

$$
\begin{aligned}
G_{1}^{S}(y, \tau)= & -\frac{h-z_{0}}{2 \pi \ell^{*}} \sum_{n=1}^{\infty} \int_{-\infty}^{\infty} \frac{d p}{\cos p} \cos (n(p-\theta)) \\
& \times f_{n}\left(\alpha_{\text {out }} \frac{h-z_{0}}{\cos p}, \alpha_{\text {out }} \sqrt{h^{2}+y^{2}}, \quad \alpha_{\text {out }} a, \alpha_{\text {in }} a\right),
\end{aligned}
$$


where

$$
\begin{aligned}
f_{n}\left(\xi_{1}, \xi_{2}, \xi_{3}, \xi_{4}\right)= & K_{n}\left(\xi_{1}\right) K_{n}\left(\xi_{2}\right) \\
& \times\left[\frac{\xi_{3} I_{n}^{\prime}\left(\xi_{3}\right) I_{n}\left(\xi_{4}\right)-\xi_{4} I_{n}\left(\xi_{3}\right) I_{n}^{\prime}\left(\xi_{4}\right)}{\xi_{3} K_{n}^{\prime}\left(\xi_{3}\right) I_{n}\left(\xi_{4}\right)-\xi_{4} K_{n}\left(\xi_{3}\right) I_{n}^{\prime}\left(\xi_{4}\right)}\right],
\end{aligned}
$$

$I_{n}$ and $K_{n}$ are modified Bessel functions, primes denote differentiation of the corresponding function with respect to its argument, $h=z+a$, and $\theta=\tan ^{-1}(y / h)$. A somewhat different result is obtained for $b<\ell^{*}$ :

$$
\begin{aligned}
G_{1}^{S}(x, y, \tau)= & -\frac{1}{2 \pi^{2}} \sum_{n=1}^{\infty} \int_{0}^{\infty} d p \cos (n \theta) \cos (p x) \\
& \times f_{n}\left(r \sqrt{p^{2}+\alpha_{\text {out }}^{2}}, r_{s} \sqrt{p^{2}+\alpha_{\text {out }}^{2}},\right. \\
a \sqrt{p^{2}+\alpha_{\text {out }}^{2}}, & \left.a \sqrt{p^{2}+\alpha_{\text {in }}^{2}}\right),
\end{aligned}
$$

where $r=\left(h^{2}+y^{2}\right)^{1 / 2}$ and $r_{s}=\left(h^{2}+y_{s}^{2}\right)^{1 / 2}$.

To satisfy the zero boundary condition in the plane $z=-z_{1}$ and thereby obtain $G_{1}^{S}$ for the experimental scheme shown in Fig. 1, we use the method of images. ${ }^{19}$ We place the images of the capillary and radiation source on the other side of the plane $z=-z_{1}$ so that the geometry of the problem would become symmetric with respect to this plane. Then the desired solution can be written as a sum of the expressions (8) or (9) and terms of the form (10) or (12) corresponding to two different capillaries and light sources.

\section{EXPERIMENTAL CONDITIONS}

A schematic diagram of the experimental apparatus is shown in Fig. 1. The sample consists of rutile $\left(\mathrm{TiO}_{2}\right)$ particles suspended in resin. A cylindrical opening with diameter $d=0.75 \mathrm{~cm}$ was made through the sample at distance $z=0.925 \mathrm{~cm}$ from one of its faces. A laminar flow of a suspension of polystyrene beads in water is maintained in the cylindrical opening by means of communicating vessels placed at different heights. The optical properties $\left(\mu_{s}^{\prime}, \mu_{a}\right)$ of the suspension are close to those of the sample material. Therefore the region inside the capillary differs from the surrounding medium only by the dynamics of the particles located in the capillary. For this reason, following the terminology of Ref. 13, we shall call this region dynamically heterogeneous.

Coherent laser radiation at wavelength $\lambda=514 \mathrm{~nm}$ and power $1 \mathrm{~W}$, generated in the $\mathrm{TEM}_{00}$ mode by an argon ion laser with a Fabry-Perot etalon placed inside the laser cavity, is injected by means of a system of mirrors and a lens into a multimode fiber-optic waveguide (core diameter 200 $\mu \mathrm{m}$, numerical aperture 0.16). The Fabry-Perot etalon inside the laser cavity gives an adequate radiation coherence length (about $3 \mathrm{~m}$ ), which is necessary in experiments on multiple scattering of light. ${ }^{20}$ Passing along the waveguide the light is incident on the surface of the sample. The light scattered from the sample is collected by means of a single-mode fiber-optic waveguide (diameter $3.1 \mu \mathrm{m}$, numerical aperture 0.13 ), which allows the fluctuations of the light intensity

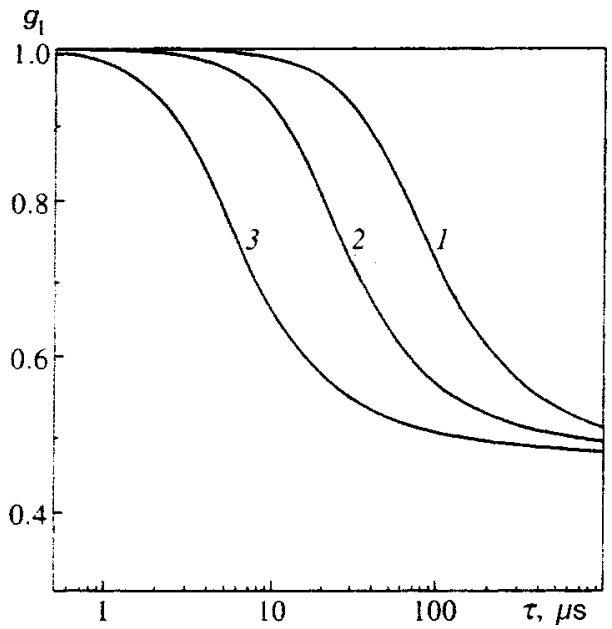

FIG. 2. Normalized time autocorrelation function of multiply scattered radiation, calculated in the diffusion approximation for the case when the radiation source and detector are arranged symmetrically relative to the capillary $\left(x=0, y=-y_{s}=2.5 \ell^{*}, z=3.5 \ell^{*}, d=3 l^{*}, z_{0}=z_{1}=\ell^{*}, \tau_{0}=6\right.$ $\left.\times 10^{-4} \mathrm{~s}\right)$. Different curves correspond to different flow velocities: $V=0.1$ (1), $0.6(2), 3(3) \mathrm{cm} / \mathrm{s}$.

within the coherence area of the scattered radiation to be recorded. Passing along the waveguide, the scattered radiation enters a photomultiplier, ${ }^{1)}$ which operates in the photon counting mode and is connected with a digital multichannel autocorrelator. $^{2)}$ The use of fiber-optic waveguides to deliver the laser radiation to the sample and to record the scattered light and the use of a digital correlator make it possible to obtain a high signal/noise ratio in the measurement process.

The measured quantity in the present experiment is the normalized autocorrelation function $g_{2}(\tau)=\langle I(t) I(t$ $-\tau)\rangle /\langle I\rangle^{2}$ of the intensity of the scattered light. The radiation scattered in the turbid medium has a Gaussian distribution, as a result of which $g_{2}(\tau)$ is related with $g_{1}(\tau)$ $=G_{1}(\tau) / G_{1}(0)$ by the Siegert relation

$$
g_{2}(\tau)=1+\beta\left|g_{1}(\tau)\right|^{2},
$$

where $0<\beta<1$ is the aperture function determined by the measurement system used. ${ }^{5,21}$ Since the sample used in the experiment does not satisfy the condition of ergodicity, the product $I(t) I(t-\tau)$ is averaged over an ensemble of realizations by the method proposed in Ref. 21 . The essence of this method is that the averaging is accomplished by moving the sample alternately in one direction and then in another relative to the stationary source and detector by means of an electric stepping motor. In our experiments the velocity of the sample motion was equal to about $50 \mu \mathrm{m} / \mathrm{s}$. The direction of motion is changed automatically, as a result of which the sample is displaced by approximately $500 \mu \mathrm{m}$ first in one and then in another direction parallel to the capillary axis.

\section{BASIC RESULTS AND DISCUSSION}

Figure 2 shows the normalized correlation function of the scattered radiation field, calculated for the case shown in Fig. 1 where the radiation is delivered and detected using thin fiber-optic waveguides $\left(b<\ell^{*}\right)$. The calculation was performed using Eqs. (9) and (12) with $\alpha_{\text {out }} \equiv 0$ and the val- 


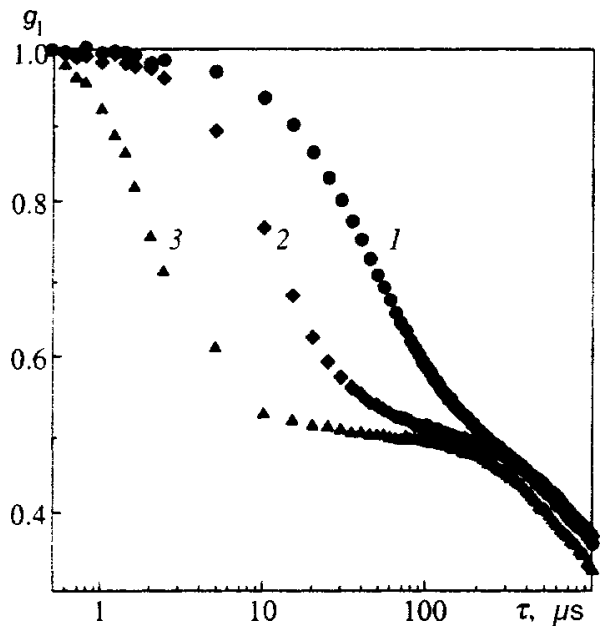

FIG. 3. Experimentally measured normalized time autocorrelation function of multiply scattered radiation for the experimental arrangement shown in Fig. 1. The radiation source and detector are arranged symmetrically with respect to the capillary $\left(x=0, y=-y_{s}=2.5 \ell^{*}\right)$. Different curves correspond to different flow velocities: $V=0.08(1), 0.62(2)$, and $3.22(3) \mathrm{cm} / \mathrm{s}$. The measurement errors fall within the size of the symbols in the figure.

ues of the other parameters close to those used in the experiment. The corresponding experimental points are presented in Fig. 3. As one can see from these figures, the section of the correlation function in the bounded range of delay times $\tau(1<\tau<400 \mu$ s under the conditions of our experiment) is most sensitive to a change in the velocity of the fluid flow inside the dynamic region; this agrees with the results obtained in Ref. 13. For $\tau<1 \mu$ s the behavior of the correlation function is determined mainly by the small but nonzero absorption of light in the medium, the absorption being the same both inside and outside the capillary. For $\tau \sim 50$ $-200 \mu \mathrm{s} g_{1}(\tau)$ tends to saturate at a constant level that is independent of the flow velocity. This fact was predicted theoretically in Ref. 12 and can be easily explained qualitatively on the basis of the correspondence between the short trajectories of photons in the medium and the long delay times $\tau:{ }^{8}$ For large $\tau$ the rate of decrease of $g_{1}(\tau)$ is determined mainly by photons with relatively short trajectories, since photons with long trajectories are now completely decorrelated. Photons with short trajectories consist mainly of photons which do not reach the capillary, and since the particles in the medium surrounding the capillary are immobile, the theoretically computed function $g_{1}(\tau)$ approaches a constant different from zero as $\tau \rightarrow \infty$. The value of this constant is determined solely by the depth at which the capillary is located. As shown in Ref. 12, for $d \gg \ell^{*}$ it can be estimated as $1-\left(z_{0}+z_{1}\right) / z \simeq 0.55$ for our sample $\left(z_{0}=\ell^{*}\right.$, $\left.z_{1}=0.7104 \ell^{*}, z=3.7 \ell^{*}\right)$. Since in Ref. 12 a laminar flow of light-scattering particles in a plane-parallel layer and not in a cylindrical capillary is studied, the agreement of this estimate with reality is satisfactory. However, as one can see from Fig. 3, the experimentally measured correlation function, though it tends to saturate at $\tau \sim 50-200 \mu \mathrm{s}$, still continues to decrease for $\tau>200 \mu \mathrm{s}$ in contrast to the function calculated theoretically (Fig. 2). This is due to effects which were neglected in our theoretical model (specifically, it is

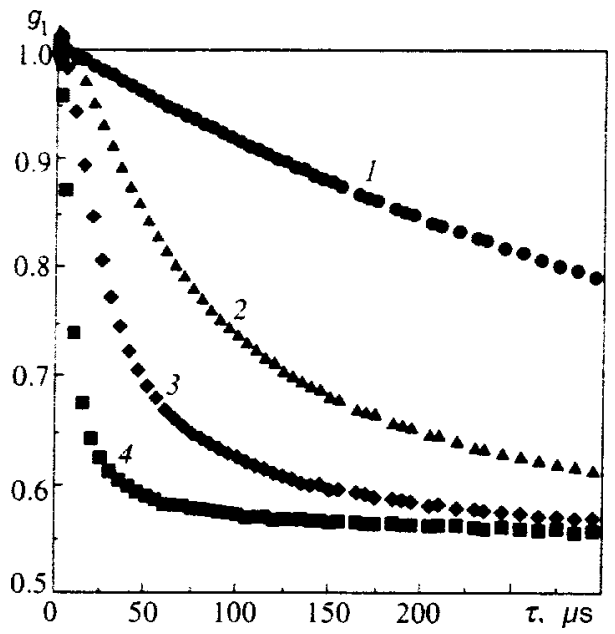

FIG. 4. Normalized time autocorrelation function of multiply scattered radiation, measured for different types of scatterer motion in the capillary: Brownian motion (1), laminar flow with velocity $V=0.08$ (2) and 0.24 (3) $\mathrm{cm} / \mathrm{s}$, turbulent flow with $V=0.88 \mathrm{~cm} / \mathrm{s}(4)$. The remaining parameters are the same as for the curves presented in Fig. 3. The measurement errors fall within the size of the symbols in the figure.

due to the method of averaging, vibration of the components of the optical system, photomultiplier noise, and so on).

We note that the theoretical and experimental results presented in Figs. 2 and 3 not only agree with one another qualitatively but they are also very close quantitatively. This attests to the fact that our theoretical approach to studying the multiple scattering of light in dynamically heterogeneous media is fully applicable to real experimental situations. Moreover, the quantitative agreement between the theoretical and experimental results makes it possible to suggest a method for determining the location and performing diagnostics of scatterer flows hidden inside strongly light-scattering media that is based on measurement and subsequent analysis of the temporal autocorrelation function of the multiplyscattered radiation. Indeed, the difference between the curves $g_{1}(\tau)$ corresponding to different average velocities $V$ of the particle flow in the capillary is quite large and can be used to measure $V$ directly. Quantitative calibration of this method of measuring the velocity can be performed on the basis of our theoretical model.

It is interesting that the method proposed for studying the dynamics of particles in turbid media can be used with equal success for different types of dynamics of scatterers in the medium. As an illustration, we present in Fig. 4 the results of measurements of the autocorrelation function of backscattered radiation for the cases of Brownian, directed, and turbulent motions of light-scattering particles in the capillary. The system of communicating vessels located at different heights was found to be inadequate for producing a turbulent fluid flow, since such a system does not make it possible to reach the critical Reynolds number on the experimental rectilinear section of the capillary. For this reason, turbulent scatterer motion in the cylindrical cavity inside the experimental sample was produced artificially with the aid of a nozzle placed at the entrance opening of the capillary.

As one can see from Fig. 4, different types of particle 
motion lead to different time dependences $g_{1}(\tau)$. This conclusion also follows from the theoretical analysis: For Brownian motion the variance of the phase difference $\left\langle\Delta \phi^{2}(\tau)\right\rangle$ for two photons scattered successively by the same particles of the medium at times separated by an interval $\tau$ is proportional to $\tau{ }^{8,9}$ For laminar and turbulent flows $\left\langle\Delta \phi^{2}(\tau)\right\rangle \propto \tau^{2},{ }^{18,22}$ but in the latter case the coefficient of proportionality is larger for the same characteristic particle velocity. Thus, there is a possibility of not only determining the location of the dynamically heterogeneous region (cylindrical capillary in our case) and estimating the average velocity of the particles inside this region but also drawing conclusions about the dominant character of the scatterer motion. We note that for the reasons explained above all curves in Fig. 4 approach the same asymptote for large $\tau$.

\section{CONCLUSIONS}

In the present paper multiple scattering of laser radiation in a randomly inhomogeneous medium with a spatially localized flow of light-scattering particles was studied. The time autocorrelation function of the light backscattered from a semi-infinite medium with a directed particle flow localized in the region of a cylindrical capillary was calculated in the diffusion approximation. The results of the theoretical analysis are in good agreement with the experimental results at the flow velocity studied. This made it possible to suggest a new method for determining the position and size of the flow region as well as the velocity of the scatterers inside this region. We note that proposed method is the only optical method that permits measuring the velocity of relatively slow (up to $10^{-2} \mathrm{~cm} / \mathrm{s}$ ) flows under conditions of multiple scattering of light in the medium. However, this method can be used only for particle flows near the boundary of the medium $\left(z<(15-20) \ell^{*}\right.$ in accordance with our theoretical calculations). This result agrees with the conclusions drawn in Refs. 12 and 13. For this reason, it can be asserted that this restriction is fundamental for turbid media and is not associated with the peculiarities of any specific measurement scheme.

Since the parameters of our sample are close to those of some biological media, ${ }^{7,15}$ our method can be used for noninvasive measurement of the characteristics of blood flow. ${ }^{14}$ Moreover, the velocity of scatterers can be measured similarly in experiments on laser acceleration of particles in dense media. ${ }^{23}$ Thus, the range of potential applications of our method is very wide.

In closing, we note that this work was made possible only by our close collaboration with D. A. Boas, B. Chance, and A. G. Yodh at the University of Pennsylvania (USA), M. Heckmeier and G. Maret at the Center de Recherches sur les Macromolecules (Strasbourg, France), and Professor R. Maynard at the Université Joseph Fourier (Grenoble, France). We thank S. S. Chesnokov, V. V. Tuchin, and A. V. Priezzhev for assistance in preparing the manuscript for publication. We are grateful to the participants of the Joint Scientific Seminar of the Department of General Physics and Wave Processes in the School of Physics at Moscow State University and the International Laser Center at Moscow State University for their interest in this work and personally to N. I. Koroteev for providing the opportunity to speak at this seminar.

${ }^{1)}$ An EMI, Gencom Inc., photomultiplier was used.

${ }^{2)} \mathrm{BI}-9000 \mathrm{AT}$, Brookhaven Instruments Corp.

${ }^{1}$ V. L. Kuz'min and V. P. Romanov, Usp. Fiz. Nauk 166, 247 (1996).

${ }^{2}$ M. P. Van Albada and A. Lagendijk, Phys. Rev. Lett. 55, 2692 (1985).

${ }^{3}$ P. E. Wolf and G. Maret, Phys. Rev. Lett. 55, 2696 (1985).

${ }^{4}$ R. Berkovits and S. Feng, 238, 135 (1994).

${ }^{5}$ A. V. Priezzhev, V. V. Tuchin, and L. P. Shubochkin, Laser Diagnostics in Biology and Medicine [in Russian], Moscow, Nauka, 1989.

${ }^{6}$ A. Yodh and B. Chance, Phys. Today 10, 34 (1995).

${ }^{7}$ V. V. Tuchin, Usp. Fiz. Nauk 167, 517 (1997).

${ }^{8}$ G. Maret and P. E. Wolf, Z. Phys. B 65, 409 (1987).

${ }^{9}$ P. M. Chaikin, D. J. Pine, D. A. Weitz, and E. Herbolzheimer, Phys. Rev. Lett. 60, 1134 (1988).

${ }^{10}$ D. A. Boas, L. E. Campbell, and A. G. Yodh, Phys. Rev. Lett. 75, 1855 (1995).

${ }^{11}$ M. Heckmeier and G. Maret, Europhys. Lett. 34, 257 (1996).

${ }^{12}$ S. E. Skipetrov and R. Maynard, Phys. Lett. A 217, 181 (1996).

${ }^{13}$ M. Heckmeier, S. E. Skipetrov, G. Maret, and R. Maynard, J. Opt. Soc. Am. A 14, 185 (1997).

${ }^{14}$ I. V. Meglinskiĭ, D. A. Boas, A. G. Yodh et al., Izv. Vyssh. Uchebn. Zaved., Ser. PND 4, 65 (1996).

${ }^{15}$ A. Ishimaru, Wave Propagation and Scattering in Random Media, Academic Press, N. Y., 1978 [Russian translation, Mir, Moscow, 1981].

${ }^{16}$ R. C. Haskell, L. V. Swaasand, T. Tsay et al., J. Opt. Soc. Am. A 11, 2727 (1994)

${ }^{17}$ D. Bicout and R. Maynard, Physica A 199, 387 (1993).

${ }^{18}$ D. Bicout and G. Maret, Physica A 210, 87 (1994).

${ }^{19}$ P. M. Morse and H. Feshbach, Methods of Mathematical Physics, McGraw-Hill, N. Y., 1953 [Russian translation, Izd. Inostr. Lit., Moscow, 1958, Vol. 1].

${ }^{20}$ T. Bellini, M. A. Glasser, N. A. Clark, and V. Degiorgio, Phys. Rev. A 44, $5215(1991)$

${ }^{21}$ J.-Z. Xue, D. J. Pine, S. T. Milner et al. Phys. Rev. A 46, 6550 (1992).

${ }^{22}$ D. J. Bicout and R. Maynard, Physica B 204, 20 (1995).

${ }^{23}$ S. E. Skipetrov, M. A. Kazaryan, N. P. Korotkov, and S. D. Zakharov, Phys. Scr. 57, 416 (1998) 